\title{
Ram pressure profiles in galaxy groups and clusters
}

\author{
Tomás E. Tecce, ${ }^{1,2 \star}$ Sofía A. Cora ${ }^{2,3,4}$ and Patricia B. Tissera ${ }^{1,2}$ \\ ${ }^{1}$ Instituto de Astronomía y Física del Espacio, C.C. 67 Suc. 28, C1428ZAA Ciudad de Buenos Aires, Argentina \\ ${ }^{2}$ Consejo Nacional de Investigaciones Científicas y Técnicas, Rivadavia 1917, C1033AAJ Ciudad de Buenos Aires, Argentina \\ ${ }^{3}$ Facultad de Ciencias Astronómicas y Geofísicas, Universidad Nacional de La Plata, Observatorio Astronómico, Paseo del Bosque S/N, B1900FWA, La \\ Plata, Argentina \\ ${ }^{4}$ Instituto de Astrofísica de La Plata, Paseo del Bosque S/N, B1900FWA La Plata, Argentina
}

Accepted 2011 June 15. Received 2011 June 15; in original form 2011 January 12

\begin{abstract}
Using a hybrid method that combines non-radiative hydrodynamical simulations with a semianalytic model of galaxy formation, we determine the ram pressure experienced by galaxies in haloes with virial masses $12.5 \leq \log \left(M_{200} / h^{-1} \mathrm{M}_{\odot}\right)<15.35$ as a function of the halocentric distance, for redshifts $0 \leq z \leq 3$. The ram pressure is calculated with a self-consistent method that uses the simulation gas particles to obtain the properties of the intergalactic medium. The ram pressure profiles obtained can be well described by beta profile models, with parameters that depend on redshift and halo virial mass in a simple fashion. The fitting formulae provided here will prove useful to include ram pressure effects into semi-analytic models based on methods that lack gas physics, such as dark-matter-only simulations or the Press-Schechter formalism.
\end{abstract}

Key words: methods: numerical - galaxies: clusters: general - galaxies: clusters: intracluster medium - galaxies: evolution - galaxies: groups: general - intergalactic medium.

\section{INTRODUCTION}

In the local Universe, the properties of galaxies depend on the environment in which they reside. The star formation rates of galaxies in denser environments such as galaxy clusters are lower than those for similar field galaxies (Kauffmann et al. 2004; Verdugo, Ziegler \& Gerken 2008) and the fraction of red galaxies at a given stellar mass increases with environmental density (Baldry et al. 2006; Martínez, Coenda \& Muriel 2008). Disc galaxies in clusters are deficient in H I when compared to similar galaxies in the field. The deficiency increases towards the cluster centre, and $\mathrm{HI}$-deficient spirals are observed to have truncated gas discs (see Boselli \& Gavazzi 2006, and references therein). These observations suggest that galaxies are transformed from blue star-forming systems into red, passive ones via physical processes that remove gas, thus suppressing the star formation in the affected galaxies.

One possible mechanism is ram pressure stripping (RPS) of galactic gas caused by the interaction with the hot, high-temperature gas of the intragroup or intracluster medium (ICM). The galaxy loses gas if the ram pressure (RP) exerted by the ICM, $P_{\text {ram }} \equiv$ $\rho_{\text {ICM }} v^{2}$, where $v$ is the velocity of the galaxy relative to the ICM, exceeds the gravitational restoring force of the galaxy (Gunn \& Gott 1972; for a recent review of observations and simulations of RPS in individual galaxies, see Roediger 2009). Prior to affecting the cold gas discs, RPS could also be responsible for the removal of the

^E-mail: tomas@iafe.uba.ar hot gas haloes of galaxies after they become satellites of a cluster (McCarthy et al. 2008; Bekki 2009).

Recent results show that RPS is not a process exclusive to cluster environments, and it can play a role in smaller systems such as galaxy groups (e.g. Rasmussen, Ponman \& Mulchaey 2006; Rasmussen et al. 2008) or for dwarf satellites of giant galaxies (e.g. McConnachie et al. 2007; Mastropietro, Burkert \& Moore 2009). Thus, a significant number of cluster galaxies could have been 'pre-processed' in the smaller systems in which they resided prior to infall into the cluster (e.g. Fujita 2004; Cortese et al. 2006).

Semi-analytic modelling of galaxy formation is a powerful approach to explore the effects of environment on galaxy properties. Such models do not require heavy computing power, and allow the exploration of a large parameter space at a fraction of the computational cost of fully self-consistent simulations. Semi-analytic models have proved very successful in reproducing several observed properties of galaxies such as the local luminosity function (see the review by Baugh 2006). RPS, however, was considered in such models only in a few cases (Okamoto \& Nagashima 2003; Lanzoni et al. 2005; Brüggen \& De Lucia 2008; Font et al. 2008). These models are based on dark matter (DM)-only simulations, and thus have to resort to analytical approximations to describe the ICM.

An alternative approach is presented by Tecce et al. (2010, hereafter T10), who combine a semi-analytic model with $\mathrm{N}$ body/hydrodynamical simulations. In this hybrid approach, the kinematical and thermodynamical properties of the ICM are provided by the gas particles of the hydrodynamical simulations. This results in a self-consistent method that does not introduce additional free parameters into the model, automatically taking into account 
local variations of the density or the velocity field. T10 find that, compared to their method, the use of analytic approximations results in overestimations of the RP larger than 50 per cent for $z>0.5$. The disadvantage of the T10 approach is that it cannot be combined with DM-only simulations, such as the widely used Millennium simulation (Springel et al. 2005), or with models that use the extended Press-Schechter (EPS) formalism (Bond et al. 1991; Bower 1991; Lacey \& Cole 1993) to determine the growth of DM haloes.

In this paper we determine fitting formulae for the RP exerted on galaxies as a function of the halocentric distance obtained by using the T10 method, in simulated galaxy groups and clusters of different masses. We show that these RP profiles can be well described using beta profile models (Cavaliere \& Fusco-Femiano 1976), with parameters depending on redshift and halo virial mass. The formulae provided here can then be used in such semi-analytic models that lack the gas physics required in the T10 approach.

\section{CONSTRUCTION OF THE RAM PRESSURE PROFILES}

To calculate the RP exerted by the intergalactic medium on satellite galaxies in groups and clusters at different redshifts, we use the hybrid model SAGRP (see T10) that combines non-radiative $\mathrm{N}$-body/smoothed particle hydrodynamics (SPH) simulations of galaxy clusters (Dolag et al. 2005, 2009) with a semi-analytic model that handles other baryonic physical processes such as gas cooling, star formation and feedback by supernovae and active galactic nuclei. The SPH simulations are resimulations of the regions around five galaxy clusters with mass $\sim 10^{14} h^{-1} \mathrm{M}_{\odot}$ (labelled g1542, g3344, g6212, g676 and g914 in Dolag et al. 2005, 2009) and three clusters with mass $\sim 10^{15} h^{-1} \mathrm{M}_{\odot}(\mathrm{g} 51, \mathrm{~g} 1$ and g8 in Dolag et al.). These correspond to a $\Lambda$ CDM cosmology with $\Omega_{\mathrm{m}}=0.3$, $\Omega_{\Lambda}=0.7$, Hubble constant $h=0.7$ (in units of $100 \mathrm{~km} \mathrm{~s}^{-1} \mathrm{Mpc}^{-1}$ ), a baryon density $\Omega_{\mathrm{b}}=0.039$ and a power spectrum normalization $\sigma_{8}=0.9$. The DM particle mass is $1.13 \times 10^{9} h^{-1} \mathrm{M}_{\odot}$ and the gas particle mass $1.69 \times 10^{8} h^{-1} \mathrm{M}_{\odot}$ (for more details see Dolag et al. 2009).

For each DM halo in the simulations, identified by means of a friends-of-friends algorithm (FoF, Davis et al. 1985), we search for all galaxies within the halo virial radius $r_{200}$, defined as the radius within which the mean mass density is $200 \rho_{\text {crit }}$. We use these galaxies as tracers of the RP at their current position, calculating for each one the RP they experience by using the properties of the surrounding gas particles (see T10 for details). We select all haloes with no contamination by boundary particles and with $\log M_{200} \geq 12.5$, where $M_{200}$ is the total mass within $r_{200}$ (in the units of $h^{-1} \mathrm{M}_{\odot}$ ). In smaller haloes the RP is very low, affecting only the smallest dwarf galaxies, and so these haloes will not be considered in this analysis.

We identify haloes in the 67 simulation snapshots available in the redshift range $0 \leq z \leq 3$. The total number of selected haloes grows steadily from $N_{\text {halo }}=29$ at $z=3$ until it reaches a peak of 155 haloes at $z \simeq 0.5$, decreasing afterwards to a final value of $N_{\text {halo }}=114$ at $z=0$. The smallest FoF haloes selected are resolved with at least $2800 \mathrm{DM}$ particles, and a similar number of gas particles. Haloes with $\log M_{200} \simeq 14$ are resolved with about $9 \times 10^{4}$ DM particles, and the most massive haloes are resolved with $10^{6}$ DM particles or more.

The positions and velocities of satellite galaxies within an FoF halo are determined by the position and velocity of the most bound DM particle of the corresponding subhalo (as done also by Brüggen \& De Lucia 2008). For those satellites whose subhalo has been

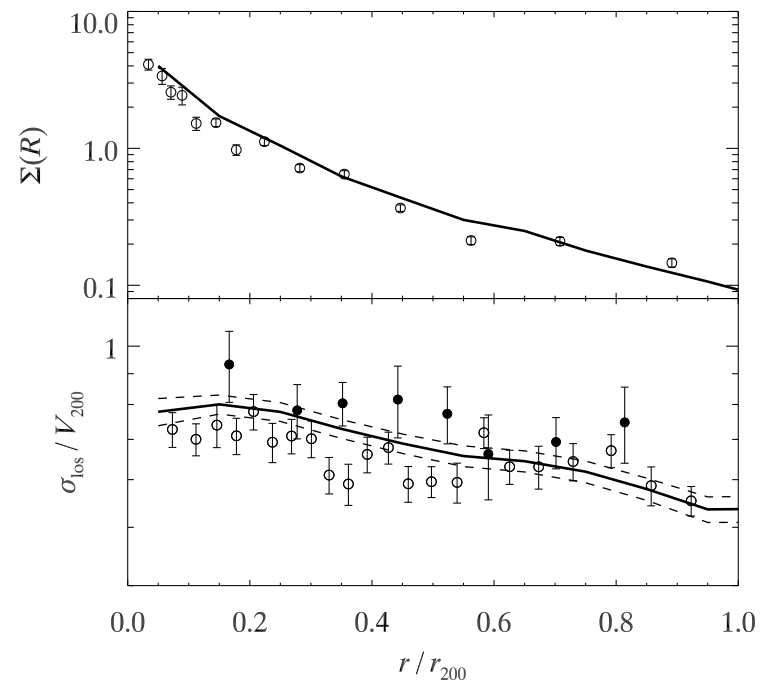

Figure 1. Top: mean projected galaxy number surface density profile for model galaxies with $M_{V}<-17$ in all haloes with $M_{200} \geq 10^{14} h^{-1} \mathrm{M}_{\odot}$ at $z=0.3$ (solid line). Open circles represent the mean observed surface density profile for CNOC survey clusters (Carlberg, Yee \& Ellingson 1997). Bottom: mean line-of-sight velocity dispersion profiles for the same haloes, at $z=0$. Filled and open circles represent, respectively, data for galaxies with and without emission lines in the ENACS clusters; from Biviano \& Poggianti (2009).

completely disrupted by the tidal forces exerted by the gravitational potential of the main halo, we use the most bound particle identified the last time that there was a subhalo. This is essentially equivalent to assuming that galaxies trace the DM. In the case of galaxy clusters, this assumption has been shown to provide a good fit to the observed galaxy number profiles out to large cluster-centric radii (see e.g. Biviano \& Girardi 2003; Gao et al. 2004).

Using DM particles as tracers for galaxies allows us to generate a population of cluster galaxies whose spatial and velocity distributions match the observed ones. The top panel in Fig. 1 shows the galaxy number surface density profile of simulated galaxies with absolute magnitude $M_{V}<-17$ in all haloes with virial mass $\geq 10^{14} h^{-1} \mathrm{M}_{\odot}$ at $z=0.3$ (solid line), compared with the observed profile for cluster galaxies in the Canadian Network for Observational Cosmology (CNOC) survey (Carlberg et al. 1997, empty circles). The density profiles for the simulations are obtained by projecting along the $x, y$ and $z$ axes in turn, considering only galaxies within $2 r_{200}$ of the cluster centre, binning the galaxies out to $2 r_{200}$ and averaging over the three projections (as in Gao et al. 2004). The mean galaxy surface density profile thus obtained agrees very well with the observational data.

The velocity dispersions that we obtain from galaxies contained within massive haloes $\left(\sigma \sim 400 \mathrm{~km} \mathrm{~s}^{-1}\right.$ for haloes with $\log M_{200} \simeq$ 14 and $\sigma \sim 900 \mathrm{~km} \mathrm{~s}^{-1}$ for haloes with $\log M_{200} \simeq 15$ ) are consistent with the mass-velocity dispersion scaling relations determined from observations (e.g. Hwang \& Lee 2008; Wojtak \& Łokas 2010). The bottom panel of Fig. 1 shows the mean line-of-sight velocity dispersion profile of the simulated galaxies at $z=0$ (solid line), constructed in the same way as the density profiles; dashed lines show the $1 \sigma$ errors. Circles show the data for ENACS clusters from Biviano \& Poggianti (2009), where filled and open symbols represent data for galaxies with and without emission lines, respectively. Although our model galaxies do not present the orbital dichotomy between early- and late-type galaxies observed (see e.g. Sodre et al. 1989; Adami, Biviano \& Mazure 1998; Biviano \& Katgert 2004), 


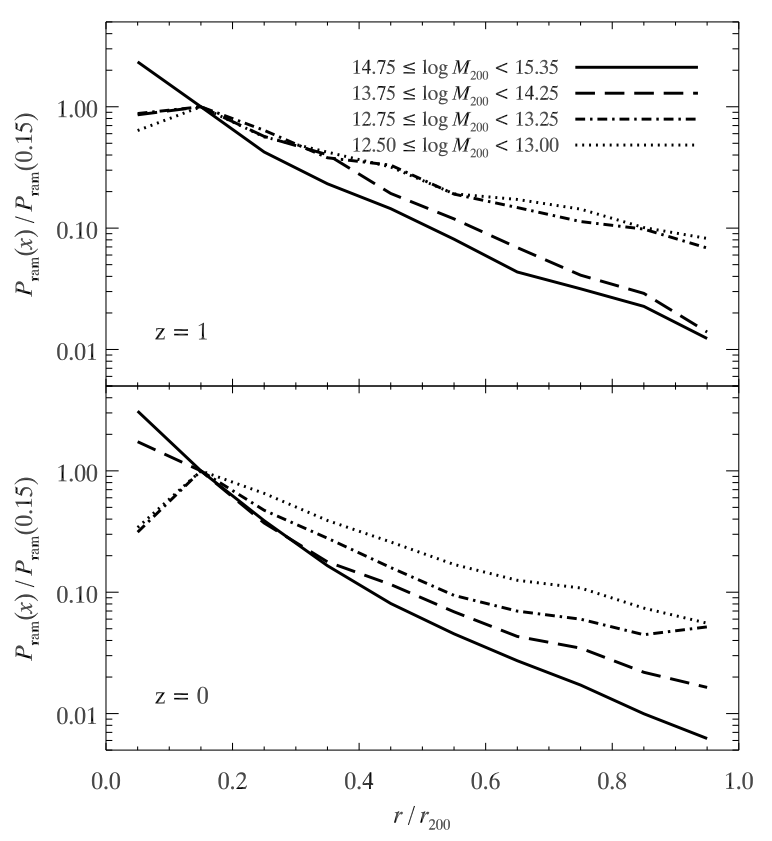

Figure 2. Combined RP profiles for haloes in four selected virial mass ranges at $z=1$ (top) and $z=0$ (bottom). Each profile is normalized to its value at $r_{N} / r_{200}=0.15$, which for the less massive haloes is equivalent to six times the gravitational softening used in the $\mathrm{N}$-body/hydrodynamical simulations.

the mean velocity dispersion profile is a very good match to the average observational trend. Therefore, we find that our choice of galaxy orbits is consistent with cluster observations, and we make the assumption that this method will provide a good approximation to the orbits of galaxies in less massive haloes as well.

In T10 it is shown that the mean RP inside a halo increases with redshift and cluster mass. Therefore, we split all the selected haloes in 10 logarithmic mass intervals in the range $12.5 \leq \log M_{200}<$ 15.35. The adopted binwidth is $\Delta \log M_{200}=0.5$. The bins overlap by half an interval, i.e. $12.5 \leq \log M_{200}<13 ; 12.75 \leq \log M_{200}<$ 13.25 and so on. By doing this we have additional points for fitting the mass dependence, in a procedure that resembles a (short-period) moving average. For each mass bin we construct a combined profile of RP versus halocentric distance by gathering the data of all the galaxies within $r_{200}$ of the corresponding haloes. We scale the halocentric galaxy distances by the $r_{200}$ of their host halo, and we determine the median RP in equally spaced radial intervals.

To determine whether the shapes of the RP profiles obtained depend on virial mass and redshift, we renormalize them to their value at $x_{N} \equiv r_{N} / r_{200}=0.15$. For the less massive haloes this is equivalent to six times the gravitational softening used in the SPH simulations. As an example, Fig. 2 shows the combined RP profiles determined for four different mass bins at $z=1$ (top panel) and $z=0$ (bottom panel). As can be seen, the combined RP profiles show different slopes for different mass intervals and times. The RP gradient is stronger in the more massive clusters, where the RP at $r=r_{200}$ is $\sim 1$ per cent of the value at $x_{N}$; in the less massive haloes, the RP at $r_{200}$ is $\sim 10$ per cent of the central value. We also find, at a given halo mass, a dependence on redshift of the normalization of the RP profiles. For example, the average RP value at $x_{N}$ varies from $\sim 1.8 \times 10^{-10} h^{2}$ dyn $\mathrm{cm}^{-2}$ at $z=1$ to $\sim 4.7 \times 10^{-10} h^{2} \mathrm{dyn} \mathrm{cm}^{-2}$ at $z=0$ for haloes with $14.75 \leq \log M_{200}<15.35$, and from $\sim 2.1 \times$ $10^{-12} h^{2}$ dyn $\mathrm{cm}^{-2}$ at $z=1$ to $\sim 4.6 \times 10^{-12} h^{2} \mathrm{dyn} \mathrm{cm}^{-2}$ at $z=0$ for haloes with $12.5 \leq \log M_{200}<13$. These trends suggest the need to

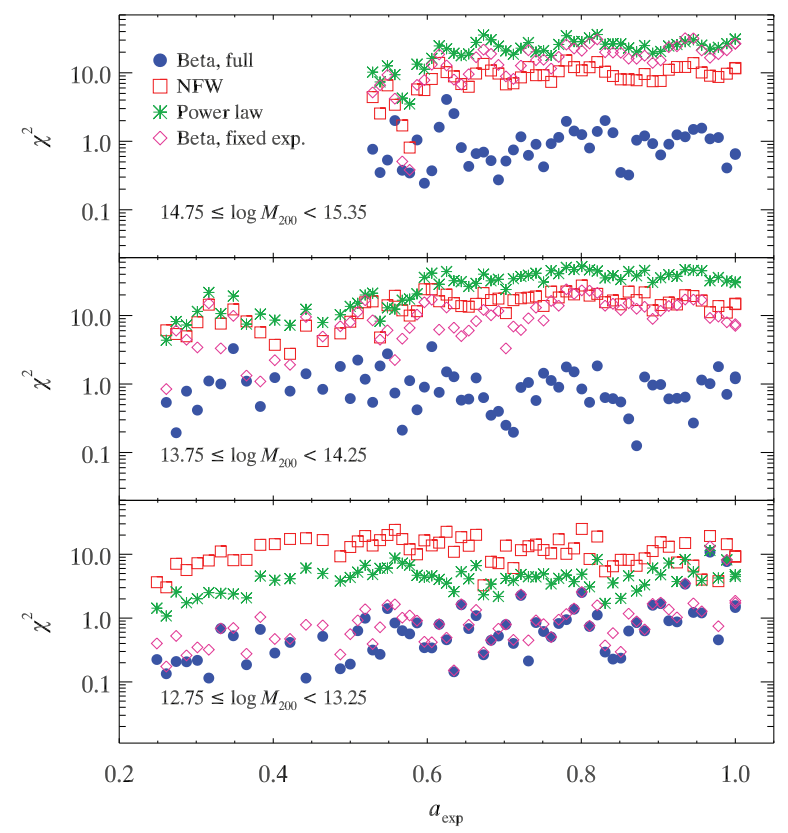

Figure 3. Chi-square goodness-of-fit estimator as a function of cosmological expansion factor $a_{\exp }$ for the four analytic profile models considered, and for three selected mass ranges: $14.75 \leq \log M_{200}<15.35$ (top), $13.75 \leq \log M_{200}<14.25$ (centre) and $12.75 \leq \log M_{200}<13.25$ (bottom). In each mass range, $\chi^{2}$ values for all the models are scaled to the average value obtained for the full beta model.

adopt a fitting function with at least two parameters, normalization and slope, both depending on halo virial mass and redshift.

\section{FITS TO THE PROFILES}

To fit the combined RP profiles obtained at each simulation snapshot for the different halo mass ranges selected, three different analytical functions are considered: a simple power law,

$P_{\text {ram }} \propto\left(r / r_{\mathrm{s}}^{P}\right)^{-\alpha}$,

a Navarro, Frenk \& White (1997, hereafter NFW) profile, which provides a good description of the density profiles of DM haloes,

$P_{\text {ram }} \propto\left(r / r_{\mathrm{s}}^{N}\right)^{-1}\left[1+\left(r / r_{\mathrm{s}}^{N}\right)\right]^{-2}$,

and a beta profile, which is commonly used to fit the density profiles of the ICM in galaxy clusters,

$P_{\text {ram }} \propto\left[1+\left(r / r_{\mathrm{s}}^{B}\right)^{2}\right]^{-3 \beta / 2}$.

In the above relations $r_{\mathrm{s}}^{P}, r_{\mathrm{s}}^{N}$ and $r_{\mathrm{s}}^{B}$ are the corresponding characteristic radii. When used to describe ICM profiles, the beta model is usually chosen to have a fixed exponent $\beta=2 / 3$. In our case, we fit one model with this fixed value, and another one where $\beta$ is allowed to vary; these models will be hereafter called fixed beta model and full beta model, respectively.

We determine the best-fitting profile of the form (1), (2) and (3) (in the last case, for both the fixed and the full beta models) for the combined RP profiles in the 10 mass intervals considered and as a function of redshift. All the fittings are carried out using the Levenberg-Marquardt technique to solve the least-squares problem (Markwardt 2009). For each fit, we compute the chi-square goodness-of-fit estimator. The chi-square values are plotted in Fig. 3 as a function of the cosmological expansion factor $a_{\exp }$ of the corresponding simulation snapshots, for three mass ranges selected 


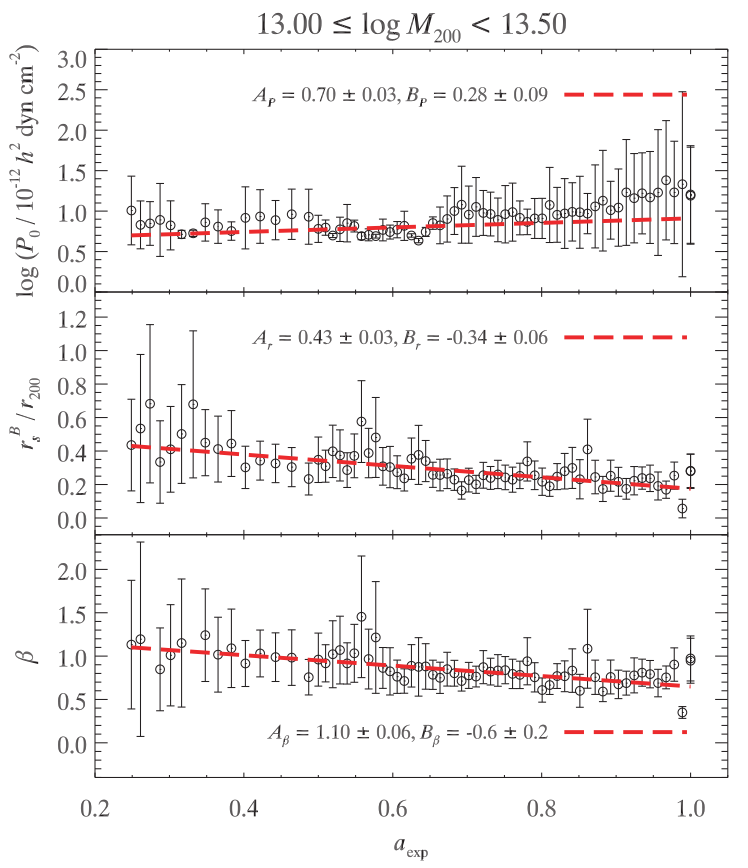

Figure 4. Evolution with time of the best-fitting parameters for the full beta model, for the mass range $13 \leq \log M_{200}<13.5$ selected as an example: logarithm of the core value $P_{0}$ (top), scale radius $r_{\mathrm{s}} / r_{200}$ (centre) and exponent $\beta$ (bottom). The dashed lines indicate linear fits to the corresponding parameters, of the form $A+B\left(a_{\exp }-0.25\right)$.

for illustration purposes only. To make the comparison easier, the chi-square values in Fig. 3 have all been normalized to the average chi-square value obtained for the full beta model, which, as can be clearly seen from the figure, provides the best-fitting profile for all times and mass ranges. Therefore, we propose as a model for the RP profiles determined with the T10 method a full beta model (3) with a core value $P_{0}$, scale radius $r_{\mathrm{s}}$ and exponent $\beta$ all depending on both virial mass and redshift:

$P_{\text {ram }}(M, z)=P_{0}(M, z)\left[1+\left(\frac{r}{r_{\mathrm{s}}(M, z)}\right)^{2}\right]^{-(3 / 2) \beta(M, z)}$.

The next step is to determine how the model parameters in (4) depend on DM halo mass and redshift. To do this we plot for each mass interval the best-fitting parameters as a function of $a_{\text {exp }}$. We find that for all the mass intervals considered, the evolution of the best-fitting parameters can be well fitted with a linear regression. As an example, Fig. 4 shows the evolution with time of the best-fitting values of $\log P_{0}, r_{\mathrm{s}}$ and $\beta$ obtained for haloes in the range $13 \leq$ $\log M_{200}<13.5$.

The zero-point and slope of the linear fits change for the different mass ranges. Hence, we propose a dependence of the model parameters of the following form:

$\log \left(\frac{P_{0}}{10^{-12} h^{2} \mathrm{dyn} \mathrm{cm}^{-2}}\right)=A_{P}+B_{P}\left(a_{\exp }-0.25\right)$,

$\frac{r_{\mathrm{s}}}{r_{200}}=A_{r}+B_{r}\left(a_{\mathrm{exp}}-0.25\right)$

$\beta=A_{\beta}+B_{\beta}\left(a_{\mathrm{exp}}-0.25\right)$, where in the above relations, the coefficients $A$ and $B$ all depend, in principle, on halo virial mass. We have chosen to express the RP values in the units of $10^{-12} h^{2}$ dyn $\mathrm{cm}^{-2}$, which is of the order of the core value of RP in the smallest haloes considered.

The fitting coefficients for the linear regressions (5) corresponding to each virial mass range are shown in Fig. 5. In all the cases, the dependence of the linear fit coefficients $A$ and $B$ on virial mass can again be very well fitted by a linear regression, chosen to be of the form $a+b\left(\log M_{200}-12\right)$. Combining the mass and redshift dependences, we finally obtain the following expressions for the coefficients in (5):

$$
\begin{aligned}
& A_{P}=(-0.8 \pm 0.1)+(1.2 \pm 0.1)\left(\log M_{200}-12\right), \\
& B_{P}=(1.2 \pm 0.2)+(-0.4 \pm 0.1)\left(\log M_{200}-12\right), \\
& A_{r}=(0.59 \pm 0.03)+(-0.14 \pm 0.02)\left(\log M_{200}-12\right), \\
& B_{r}=(-0.44 \pm 0.06)+(0.12 \pm 0.04)\left(\log M_{200}-12\right),
\end{aligned}
$$

$A_{\beta}=0.92 \pm 0.08$,

$B_{\beta}=-0.4 \pm 0.1$.

To illustrate the potential of our model, we have run a modified version of SAGRP in which we replaced the RP calculation using the gas particles from the underlying simulations by the fitting formulae determined in this work. We will hereafter refer to this modified model as SAGRP-F. In SAGRP-F, as an approximation we set the RP equal to zero for all galaxies if $z>3$, and for $z=3$ onwards we determine, for all satellite galaxies in each halo, the distance to the halo central galaxy and then the corresponding RP using (4), (5) and (6). We compare the results of SAGRP-F with those from SAGRP and also with SAGRP-A, a model where the RP is calculated analytically by using an NFW profile for the ICM density and assuming the ICM to be in a hydrostatical equilibrium (see T10 for details).

Fig. 6 shows the fraction of satellite galaxies with stellar mass $\geq 10^{9} h^{-1} \mathrm{M}_{\odot}$ that have completely lost their cold gas mass as a function of halocentric distance, for the three different models runs: SAGRP (solid lines), SAGRP-A (dot-dashed lines) and SAGRP-F (dashed lines). Results are shown grouping the halo masses in three separate bins: $14.5 \leq \log M_{200} \leq 15.5,13.5 \leq \log M_{200}<14.5$ and $12.5 \leq$ $\log M_{200}<13.5$ (top, centre and bottom rows, respectively), and for three selected redshifts: $z=1$ (left column), $z=0.5$ (centre column) and $z=0$ (right column).

As already shown in T10, calculating the RP by using an analytical approximation is a good match for the most massive clusters at $z \sim 0$, but it grossly overestimates the RP for $\log M_{200} \lesssim 14$ at $z>0$. On the other hand, Fig. 6 clearly shows that our best-fitting formulae provide an excellent match to the results of the self-consistent $\mathrm{RP}$ estimation of SAGRP, matching the slope and evolution of the fractions in all the mass ranges and at all the redshifts considered. For the two most massive ranges considered (top and centre rows in Fig. 6), the SAGRP-F model appears to systematically underestimate the RP; however, the difference is within the $1 \sigma$ errors for the models. 

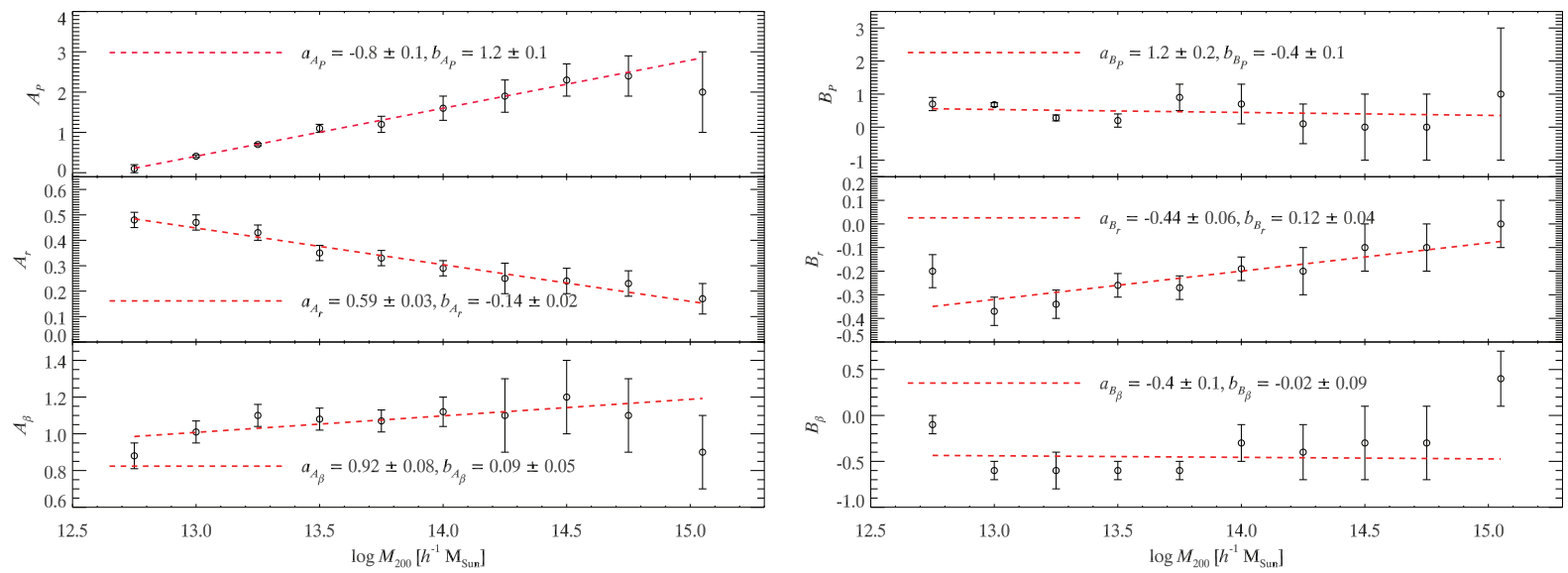

Figure 5. Values of the parameters obtained when fitting the time evolution of the best-fitting parameters for the full beta model (4) with a straight line $A+B\left(a_{\exp }-0.25\right)$, plotted as a function of halo virial mass: $\log P_{0}$ (top panels), $r_{\mathrm{s}} / r_{200}$ (centre) and $\beta$ (bottom). Left-hand and right-hand panels show the corresponding values for the coefficients $A$ and $B$, respectively. In all cases, the mass dependence of the coefficients can be fitted again with a linear regression of the form $a+b\left(\log M_{200}-12.0\right)$, shown with a dashed line.

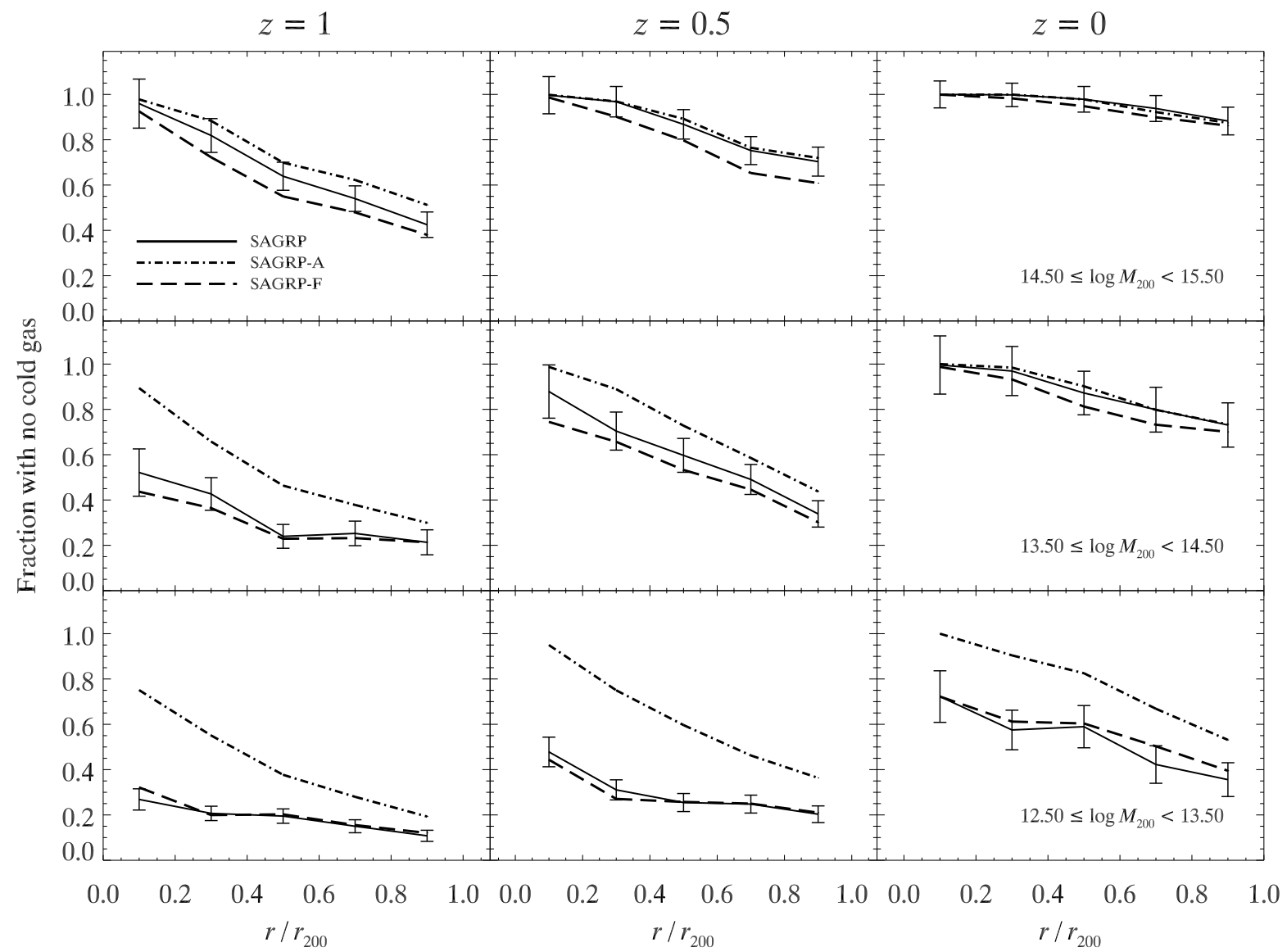

Figure 6. Fraction of galaxies with stellar mass $\geq 10^{9} h^{-1} \mathrm{M}_{\odot}$ that have completely lost their cold gas as a function of halocentric distance, for three different models: SAGRP, which determines the RP from the information provided by the gas particles in the underlying simulations (solid lines); sAGRP-A, which calculates the RP by using an analytical approximation (dot-dashed lines); and SAGRP-F, which uses the best-fitting formulae determined in this work (dashed lines). Results are presented for $z=1,0.5$ and 0 (left, centre and right columns, respectively) and for haloes in three different mass ranges: $14.5 \leq \log M_{200} \leq 15.5$ (top row), $13.5 \leq \log M_{200}<14.5$ (centre row) and $12.5 \leq \log M_{200}<13.5$ (bottom row). Error bars show the $1 \sigma$ errors for the SAGRP model.

\section{CONCLUSIONS}

Using a hybrid method that combines a semi-analytic model of galaxy formation with cosmological hydrodynamical simulations, which takes advantage of the extra kinematical and thermodynam- ical information provided by the simulation gas particles, we have determined the RP as a function of halocentric distance for DM haloes with virial masses $12.5 \leq \log M_{200}<15.35$, for redshifts in the range $0 \leq z \leq 3$. The RP profiles found can be well described by beta models, with parameters that depend on virial mass and 
redshift in a relatively simple fashion. The resulting prescriptions can be used to include the effect of RPS in semi-analytic models in which the growth of DM haloes is determined either from cosmological $N$-body simulations that do not include gas particles, or with an approach based on the EPS formalism (e.g. Sheth \& Tormen 2002; Cole et al. 2008; Neistein, Macciò \& Dekel 2010).

In our best-fitting formulae for the RP profiles given by (4), (5) and (6), we note that whereas the core RP value $P_{0}$ and scale radius $r_{\mathrm{s}}$ depend on both halo virial mass and redshift, the exponent $\beta$ depends only on time. We have checked this by running several different variants of the SAGRP-F model, by including in equations (6e) and (6f) a mass-dependent term similar to those in the relations for the other parameters, and by varying the coefficients $b_{A_{\beta}}$ and $b_{B_{\beta}}$ (see Fig. 5) within the range of errors obtained for them. In all cases, we find that the smallest difference between SAGRP and SAGRP-F is obtained when $\beta$ is assumed to be independent of virial mass.

The results presented in this work extend those of T10 and Brüggen \& De Lucia (2008), who focused on the most massive clusters with $\log M_{200} \gtrsim 14$, by also considering the RP profiles in less massive galaxy groups. The gradient of RP becomes steeper as the virial mass increases; in the massive clusters, the RP at the core is $\sim 100$ times higher than at $r=r_{200}$, whereas in galaxy group-sized haloes the RP at $r_{200}$ is about 10 per cent of the central value. On the other hand, the RP on the outskirts of $\log M_{200}=15$ clusters is $\sim 5 \times 10^{-12} h^{2} \mathrm{dyn} \mathrm{cm}^{-2}$ at $z=0$, of the same order of magnitude as the RP experienced by galaxies in the cores of haloes with $\log M_{200}=13.5$ at the same epoch. Using SPH simulations, Roediger \& Brüggen (2006) find that such levels of RP can remove about a quarter of the total gas of a spiral galaxy with mass $\sim 2 \times 10^{11} \mathrm{M}_{\odot}$. One concludes that dwarf galaxies with stellar mass $\lesssim 10^{9} \mathrm{M}_{\odot}$ in groups of all masses are very likely to experience gas loss by RPS, at least in the local Universe when the RP levels are higher in all haloes.

The fits obtained in this work were determined by using a set of resimulations including the gas physics of the regions surrounding eight massive galaxy clusters, extracted originally from a DM-only cosmological simulation. These regions are large enough to contain a fair sample of haloes in the mass range considered, free of contamination from boundary particles. Haloes in the lowest mass bin are resolved with at least $2800 \mathrm{DM}$ particles, and the most massive haloes are resolved with $10^{6} \mathrm{DM}$ particles or more. However, we are restricted to only a few massive clusters (there are only three haloes in the most massive bin), and this increases the relative error in the fitting parameters.

The RPS depends on the orbits of the satellite galaxies. There are several different approaches to determining the orbits of satellites in a semi-analytic model (see e.g. Lanzoni et al. 2005; Cora 2006; Font et al. 2008). As in Brüggen \& De Lucia (2008), we track the orbital evolution of satellite galaxies within haloes by following the most bound DM particle of their subhalo. We have shown that the method chosen results in positions and velocities of cluster galaxies which are in a good agreement with the mean observed trends, although in the case of the velocity dispersion profiles the dichotomy between early- and late-type galaxies is not seen in the model galaxies. This may point to missing physics in the models, since it is still unclear which process is responsible for the observed differences (see Biviano \& Poggianti 2009).

The particular profile fits obtained could also depend on the method chosen for the hydrodynamical calculation in the simulations. For example, the non-radiative SPH simulations used in this work may suffer from an artificially suppressed turbulence (see Dolag et al. 2005 and Agertz et al. 2007). Use of simulations carried out using a grid-based method, for example, could result in an increased turbulence in the ICM and lead to a larger scatter in the profiles, as has already been noted in T10. We intend to explore this issue in the future, as larger simulations including different implementations for the gas physics become available to us.

Notwithstanding the above considerations, within the current knowledge and taking into account the commonly used hypotheses, our best-fitting formulae capture remarkably well the effects of $\mathrm{RP}$, as given by the hybrid approach of T10, on galaxies in different DM halo masses at different redshifts, and provide a significant improvement over the analytical approximations used so far to estimate RPS effects.

\section{ACKNOWLEDGMENTS}

The authors thank the referee for useful comments. They would also like to thank Klaus Dolag for making the hydrodynamical simulations available to them, and Ho Seong Hwang and Andrea Biviano for providing observational data. This work was partially supported by PICT 245 Max Planck (2006) and PIP 0305/09 (CONICET).

\section{REFERENCES}

Adami C., Biviano A., Mazure A., 1998, A\&A, 331, 439

Agertz O. et al., 2007, MNRAS, 380, 963

Baldry I. K., Balogh M. L., Bower R. G., Glazebrook K., Nichol R. C., Bamford S. P., Budavari T., 2006, MNRAS, 373, 469

Baugh C. M., 2006, Rep. Progress Phys., 69, 3101

Bekki K., 2009, MNRAS, 399, 2221

Biviano A., Girardi M., 2003, ApJ, 585, 205

Biviano A., Katgert P., 2004, A\&A, 424, 779

Biviano A., Poggianti B. M., 2009, A\&A, 501, 419

Bond J. R., Cole S., Efstathiou G., Kaiser N., 1991, ApJ, 379, 440

Boselli A., Gavazzi G., 2006, PASP, 118, 517

Bower R. G., 1991, MNRAS, 248, 332

Brüggen M., De Lucia G., 2008, MNRAS, 383, 1336

Carlberg R. G., Yee H. K. C., Ellingson E., 1997, ApJ, 478, 462

Cavaliere A., Fusco-Femiano R., 1976, A\&A, 49, 137

Cole S., Helly J., Frenk C. S., Parkinson H., 2008, MNRAS, 383, 546

Cora S. A., 2006, MNRAS, 368, 1540

Cortese L., Gavazzi G., Boselli A., Franzetti P., Kennicutt R. C., O’Neil K., Sakai S., 2006, A\&A, 453, 847

Davis M., Efstathiou G., Frenk C. S., White S. D. M., 1985, ApJ, 292, 371

Dolag K., Vazza F., Brunetti G., Tormen G., 2005, MNRAS, 364, 753

Dolag K., Borgani S., Murante G., Springel V., 2009, MNRAS, 399, 497

Font A. S. et al., 2008, MNRAS, 389, 1619

Fujita Y., 2004, PASJ, 56, 29

Gao L., White S. D. M., Jenkins A., Stoehr F., Springel V., 2004, MNRAS, 355,819

Gunn J. E., Gott J. R. I., 1972, ApJ, 176, 1

Hwang H. S., Lee M. G., 2008, ApJ, 676, 218

Kauffmann G., White S. D. M., Heckman T. M., Ménard B., Brinchmann J., Charlot S., Tremonti C., Brinkmann J., 2004, MNRAS, 353, 713

Lacey C., Cole S., 1993, MNRAS, 262, 627

Lanzoni B., Guiderdoni B., Mamon G. A., Devriendt J., Hatton S., 2005, MNRAS, 361, 369

Markwardt C. B., 2009, in Bohlender D. A., Durand D., Dowler P., eds, ASP Conf. Ser. Vol. 411, Astronomical Data Analysis Software and Systems XVIII. Astron. Soc. Pac., San Francisco, p. 251

Martínez H. J., Coenda V., Muriel H., 2008, MNRAS, 391, 585

Mastropietro C., Burkert A., Moore B., 2009, MNRAS, 399, 2004

McCarthy I. G., Frenk C. S., Font A. S., Lacey C. G., Bower R. G., Mitchell

N. L., Balogh M. L., Theuns T., 2008, MNRAS, 383, 593 
McConnachie A. W., Venn K. A., Irwin M. J., Young L. M., Geehan J. J., 2007, ApJ, 671, L33

Navarro J. F., Frenk C. S., White S. D. M., 1997, ApJ, 490, 493

Neistein E., Macciò A. V., Dekel A., 2010, MNRAS, 403, 984

Okamoto T., Nagashima M., 2003, ApJ, 587, 500

Rasmussen J., Ponman T. J., Mulchaey J. S., 2006, MNRAS, 370, 453

Rasmussen J., Ponman T. J., Verdes-Montenegro L., Yun M. S., Borthakur S., 2008, MNRAS, 388, 1245

Roediger E., 2009, Astron. Nachr., 330, 888

Roediger E., Brüggen M., 2006, MNRAS, 369, 567
Sheth R. K., Tormen G., 2002, MNRAS, 329, 61

Sodre L., Jr, Capelato H. V., Steiner J. E., Mazure A., 1989, AJ, 97, 1279

Springel V. et al., 2005, Nat, 435, 629

Tecce T. E., Cora S. A., Tissera P. B., Abadi M. G., Lagos C. D. P., 2010, MNRAS, 408, 2008

Verdugo M., Ziegler B. L., Gerken B., 2008, A\&A, 486, 9

Wojtak R., Łokas E. L., 2010, MNRAS, 408, 2442

This paper has been typeset from a $\mathrm{T}_{\mathrm{E}} \mathrm{X} / \mathrm{L} \mathrm{T} \mathrm{T}_{\mathrm{E}} \mathrm{X}$ file prepared by the author. 